\title{
Bibliometrie
}

Andreas Barth* und Werner Marx

\section{Referenzjahrgangs-Spektroskopie: Eine bibliometrische Methode zur Untersuchung der historischen Wurzeln von Forschungsfeldern}

DOI 10.1515/iwp-2017-0006

Zusammenfassung: In der Forschungsevaluierung wird die Wirkung (Impact) wissenschaftlicher Publikationen häufig über die Anzahl ihrer Zitierungen mit Hilfe quantitativer (bibliometrischer) Verfahren gemessen. Bibliometrische Daten unterstützen und ergänzen die Arbeit der Fachgutachter. Alternativ kann man aber auch die Fachliteratur eines ausgewählten Forschungsfeldes darauf hin untersuchen, welche Arbeiten, Autoren oder Zeitschriften besonders häufig referenziert (zitiert) wurden. Von besonderem Interesse für den Ursprung und die Entwicklung eines Forschungsfeldes ist es zu erfahren, welche Arbeiten im Verlaufe der Zeit einen besonders starken Beitrag zu dessen Entstehung geleistet haben. Damit eröffnet sich die Möglichkeit, die Entwicklung von Forschungsfeldern zeitlich $\mathrm{zu}$ verfolgen und das Gewicht einzelner Arbeiten $\mathrm{zu}$ quantifizieren. Die Daten beruhen auf der Gesamtheit der Fachkollegen und liefern z.B. Wissenschaftshistorikern die potenziell wichtigen Beiträge, die sie bei ihrer Arbeit berücksichtigen sollten, um Unvollständigkeiten und Verzerrungen zu vermeiden. Die entsprechende bibliometrische Methode ist die Referenzjahrgangs-Spektroskopie (RPYS: Reference Publication Year Spectroscopy). Mit der Anwendung dieser Methode macht man sich die Tatsache zunutze, dass ungewöhnlich hoch zitierte Arbeiten im RPYS-Spektrum als markante Spitzen (Peaks) in den betreffenden Publikationsjahren auftreten. Für die Auswertung wurde ein Programm namens Cited References Explorer (CRExplorer) entwickelt. In dieser Arbeit möchten wir die RPYS-Methode an Hand von zwei ausgewählten Beispielen vorstellen und deren Anwendung auf Themen der Wissenschaftsgeschichte diskutieren. Im Ergebnis unserer

*Kontaktperson: Dr. Andreas Barth, FIZ Karlsruhe, Leibniz-Institut für Informationsinfrastruktur, Hermann-von-Helmholtz-Platz 1, 76344 Eggenstein-Leopoldshafen, E-Mail: andreas.barth@fiz-karlsruhe.de, www.fiz-karlsruhe.de, orcid.org/0000-0002-1626-5121

Dr. Werner Marx, Max-Planck-Institut für Festkörperforschung, Heisenbergstraße 1, 70569 Stuttgart, E-Mail:w.marx@fkf.mpg.de, http://www.fkf.mpg.de/ivs, orcid.org/0000-0002-1763-5753
Analyse spiegelt sich das Wissen der Gesamtheit der Fachkollegen wider: Es finden sich alle in der jeweiligen wissenschaftshistorischen Fachliteratur genannten wesentlichen frühen Beiträge zum entsprechenden Forschungsfeld. Die Methode funktioniert ohne jedwede weiteren Annahmen und vermeidet damit z. B. eine subjektive Auswahl, die im Falle einzelner Autoren von wissenschaftshistorischen Abhandlungen unvermeidlich ist.

Deskriptoren: Bibliometrie, Wissenschaftsgeschichte, Referenzjahrgangs-Spektroskopie

Reference publication year spectroscopy: A bibliometric method for the analysis of the historical roots of research fields

Abstract: In research evaluations, the impact of scientific publications is often measured by the number of their citations with the help of quantitative (bibliometric) methods. Bibliometric data support and complement the work of experts. Alternatively, one can also examine the literature of a chosen research field with respect to most frequently cited publications, authors, and journals. It is particularly interesting for the origin and development of a research field to understand which publications have, in the course of time, made an outstanding contribution to its development. This opens up an opportunity to pursue the development of research fields chronologically and to quantify the weight of individual publications. The data are based on the scientific community as a whole and they provide, e.g. for science historians, the potentially important contributions they should consider in their scientific work in order to avoid incompleteness and distortions. The appropriate bibliometric method is called Reference Publication Year Spectroscopy (RPYS) and it makes use of the fact that very frequently cited papers occur as prominent peaks in the corresponding publication years. A program called Cited References Explorer (CRExplorer) has been developed for the final analysis. In this paper, we would like to present the RPYS method on the basis of two selected examples and discuss its application to topics of the history of science. 
The result of our analysis reflects the knowledge of the scientific community as a whole: one can find all the essential early contributions cited in the corresponding scientific historical literature regarding a specific research field. The method works without any additional assumptions and thus avoids, e.g. a personal selection, which is inevitable in the case of individual authors of scientific historical publications.

Descriptors: Bibliometry, History of science, Reference publication year spectroscopy

\section{La spectroscopie de l'année des références aux publicati- ons: Une méthode bibliométrique pour l'analyse des raci- nes historiques des domaines de recherche}

Résumé: Dans l'évaluation de la recherche, l'impact des publications scientifiques est fréquemment mesuré par le nombre de leurs citations à l'aide de procédés quantitatifs bibliométriques. Les données bibliométriques soutiennent et complètent le travail des experts d'un domaine. Pourtant, comme alternative, on peut aussi examiner la littérature spécialisée d'un domaine choisi afin de découvrir quels ouvrages et auteurs ou quelles revues étaient particulièrement fréquemment référencés (cités). Pour comprendre l'origine et le développement d'un domaine de recherche, il est très intéressant de connaître les ouvrages qui ont, au cours du temps, contribué particulièrement à sa formation. Avec cela s'ouvre la possibilité de poursuivre chronologiquement le développement des domaines de recherche et de quantifier l'importance d'ouvrages particuliers. Les données reposent sur l'ensemble des collègues du domaine et offrent, par exemple aux historiens scientifiques, les contributions potentiellement importantes qu'ils devraient prendre en considération pour éviter que leurs travaux soient incomplets ou déformés. La méthode bibliométrique appropriée est celle de la spectroscopie de l'année des références aux publications (RPYS: Reference Publication Year Spectroscopy). Par l'emploi de cette méthode on tire profit du fait que les ouvrages extraordinairement souvent cités apparaissent, dans le spectre de la RPYS, sous la forme de pics (peaks) marqués dans les années de publication correspondantes. Pour leur évaluation, un programme du nom de Cited Reference Explorer (CRExplorer) était développé. Dans cette étude, nous voulons présenter la méthode RPYS à l'aide de deux exemples choisis et discuter de son application aux thèmes de l'histoire des sciences. Le résultat de notre analyse reflète le savoir de l'ensemble des collègues du domaine, c'est-àdire, pour chaque domaine de recherche s'y trouvent toutes les premières contributions essentielles qui sont indiquées dans chaque littérature spécialisée relative à l'his- toire des sciences. La méthode fonctionne sans aucune autre condition et évite ainsi, par exemple, un choix subjectif, qui est inévitable dans le cas de quelques auteurs d'études relatives à l'histoire des sciences.

Descripteurs: Bibliométrie, Histoire des sciences, Spectroscopie de l'année de publication des références

\section{Einleitung}

Wissenschaftlicher Fortschritt resultiert aus den Forschungsaktivitäten einer Vielzahl von Wissenschaftlern, oft über lange Zeiträume hinweg. Selbst für Experten eines Forschungsfeldes oder für Wissenschaftshistoriker ist es nicht leicht, Entdecker zu benennen und die für die weitere Entwicklung eines Forschungsfeldes entscheidenden Arbeiten festzumachen. Die historischen Wurzeln bzw. intellektuellen Ursprünge einer Methode oder Theorie verlieren sich oft im Dunkel der Vorgeschichte. Vorläufer, Entdecker und Unterstützer sind kaum auseinander zu halten. Die entscheidenden Beiträge stammen jedoch zumeist von einer überschaubaren Anzahl von Forschern und wurden in verhältnismäßig wenigen Veröffentlichungen publiziert. Mittels einer statistischen Auswertung der in der gesamten Fachliteratur eines Forschungsfeldes enthaltenen Referenzen (Literaturverweise, auch Zitierungen, Zitate oder Zitationen genannt) können solche Arbeiten jedoch als potenziell in Betracht zu ziehende wichtige Beiträge ermittelt werden. Die Bibliometrie als quantitative Methode zur Analyse von Fachliteratur und deren Zitierungen ist vor allem durch ihren Einsatz in der Forschungsevaluierung bekannt geworden. Sie bietet inzwischen aber auch die passenden Methoden und Werkzeuge, um in der Wissenschaftsgeschichte eingesetzt zu werden.

Der Begriff Bibliometrie wurde von Alan Pritchard (1969) durch die Definition geprägt: "bibliometrics is the application of mathematical and statistical methods to books and other media of communication". Inzwischen hat sich die Bibliometrie als eine eigenständige interdisziplinäre Wissenschaft etabliert. Sie basiert auf der statistischen Analyse von Metadaten zu wissenschaftlichen Publikationen oder Patentdokumenten und deren Zitierungen. Die wichtigsten Informationsquellen sind bibliographische Datenbanken, die zusätzlich die Literaturzitate (Referenzen) enthalten. Dazu gehören vor allem die disziplinübergreifenden Datenbanken Web of Science (WoS) von Clarivate Analytics (vormals Thomson Reuters) und Scopus von Elsevier. Aber auch fachspezifische Datenbanken wie CAplus (Chemie) von Chemical Abstracts oder zbMATH (Mathematik) von FIZ Karlsruhe können als Datengrundlage für bi- 


\begin{tabular}{|lll|}
\hline Bibliometrie im Allgemeinen & Vorwärtszitierungen & $\begin{array}{l}\text { Wie oft wurde eine ausgewählte } \\
\text { Arbeit, ein Autor, ein Institut, eine } \\
\text { Zeitschrift etc. insgesamt zitiert? }\end{array}$ \\
$\begin{array}{l}\text { Reference Publication Year } \\
\text { Spectroscopy (RPYS) }\end{array}$ & Rückwärtszitierungen & $\begin{array}{l}\text { Welche Arbeiten, Autoren, } \\
\text { Zeitschriften etc. werden innerhalb } \\
\text { eines Forschungsfeldes besonders } \\
\text { häufig zitiert? }\end{array}$ \\
\hline
\end{tabular}

Abbildung 1: Vergleich von allgemeiner Bibliometrie und der Reference Publication Year Spectroscopy (RPYS).

bliometrische Analysen herangezogen werden. Unsere Analyse basiert auf Recherchen in der Datenbank WoS, allerdings können derartige Untersuchungen auch in der entsprechenden Datenbank SCISEARCH in STN ausgeführt werden. Die Datenbank WoS enthält alle Publikationen (citing papers) aus ca. 9.000 Kernzeitschriften zusammen mit allen darin enthaltenen Referenzen (cited references). Der Erfassungszeitraum der Publikationen beginnt 1900 und beschränkt sich auf die 9.000 Kernzeitschriften, während die Referenzen alle zitierten Arbeiten umfassen, unabhängig vom Erscheinungsjahr, dem Fachgebiet und der Publikationsart (Buch, Zeitschriftenartikel, etc.).

Neben ihrem Einsatz bei der Erstellung von Daten zum Zwecke der Forschungsevaluierung ist die Bibliometrie aber auch selbst eine eigene Forschungsdisziplin geworden. Schwerpunkte der bibliometrischen Forschung sind (1) die Entwicklung von neuen bibliometrischen Gesetzen und Bewertungsindikatoren (bibliometrics for bibliometricians), (2) Themen im Umfeld der Forschungsevaluation (bibliometrics for science policy and management) und (3) die Analyse von wissenschaftlichen Problemen mit Hilfe von bibliometrischen Methoden und Werkzeugen (bibliometrics for scientific disciplines). Wichtige Pioniere dieser Disziplin waren u. a. Derek John de Solla-Price (1922-1983) (de Solla-Price 1974) und Eugene Garfield (1925-) (Garfield 1979); zusammenfassende Darstellungen zur Bibliometrie findet man z. B. bei Glänzel (2003) oder Havemann (2009). Eine Übersicht zum Einsatz der Bibliometrie in der Forschungsevaluierung liefert Moed (2005).

Eine Vielzahl von bibliometrischen Untersuchungen hat gezeigt, dass Fachartikel eine sehr unterschiedliche Resonanz in Form von Zitierungen erfahren und damit unterschiedlich stark zum wissenschaftlichen Fortschritt beitragen: Verhältnismäßig wenige Arbeiten erfahren eine große Aufmerksamkeit und viele Arbeiten werden später kaum oder gar nicht mehr erwähnt. Diese „schiefe“ Verteilung der Zitierungen über die Publikationen kann man sich beim Auffinden der für die Entstehung und Fortentwicklung eines Forschungsfeldes relevanten Publikationen zunutze machen.

\section{Die RPYS-Methode}

Vor einigen Jahren wurde die „Reference Publication Year Spectroscopy“ (RPYS) ${ }^{1}$ als eine neue bibliometrische Methode eingeführt (Marx et al.,2014). Sie ermöglicht die Identifikation von besonders häufig zitierten Arbeiten innerhalb eines Forschungsfeldes - und damit von historisch besonders relevanten wissenschaftlichen Publikationen. Im Allgemeinen nutzt die Bibliometrie die Anzahl der Zitierungen von Arbeiten als Maß für ihre Wirkung (Impact), dokumentiert durch die Referenzen in den Publikationen der Fachkollegen (Bornmann \& Marx 2014). Wirkung als Maß für Bedeutung wird dabei als messbarer Anteil von Qualität aufgefasst. Zitierungsdaten beruhen normalerweise auf den zu bewertenden Publikationen und deren Zitierungen über alle Disziplinen hinweg. Man kann aber einen Perspektivwechsel vornehmen und die Referenzen analysieren, die innerhalb eines klar abgegrenzten Forschungsfeldes in den dazugehörigen Publikationen zitiert wurden. Man erfährt dabei z.B., welche Arbeiten, Autoren, Zeitschriften usw. für die betreffenden Fachkollegen besonders wichtig waren und deshalb häufig zitiert wurden. Während die Bibliometrie im Allgemeinen auf den Vorwärtszitierungen basiert, macht sich die RPYS-Methode die Rückwärtszitierungen zunutze (s. Abb. 1).

Wenn man die Publikationsjahre der zitierten Referenzen analysiert, so findet man, dass in manchen Jahren besonders viele Zitierungen vorkommen. Die Auftragung der Häufigkeiten der Referenzjahrgänge auf der Zeitachse liefert in aller Regel ausgeprägte Spitzen (Peaks), die durch überdurchschnittlich hohe Zitierungen einzelner Arbeiten zustande kommen. Diese Arbeiten waren für die Fachkollegen so wichtig, dass sie über die Jahre hinweg immer wieder zitiert wurden. Die Arbeiten des gleichen

1 Der Name der Methode „Reference Publication Year Spectroscopy“ (deutsch: Referenzjahrgangs-Spektroskopie) wurde aufgrund der Analogie zur Spektroskopie in der Chemie gewählt. Wenn man die Häufigkeiten der Zitierungen (cited references) nach dem Reference Publication Year (RPY) aufträgt, erhält man eine grafische Darstellung mit markanten Peaks, die an ein Spektrum erinnert. 
Referenzjahrgangs sind hinsichtlich ihrer Zitierhäufigkeiten unmittelbar miteinander vergleichbar, weil sie dasselbe Alter aufweisen und in der Regel auch aus dem gleichen Fachgebiet stammen. Bedingt durch das rapide Wachstum der Anzahl wissenschaftlicher Publikationen (und damit auch der zitierten Referenzen) erscheinen die Peaks zur Gegenwart hin (etwa ab dem Jahr 1990) normalerweise immer weniger ausgeprägt. Da oft mehrere häufig zitierte Publikationen im selben Jahr erschienen sind, wird das Hervortreten einzelner Arbeiten unterdrückt. Aus diesen Gründen ist die RPYS-Methode vor allem zur Analyse älterer Referenzen und damit der historischen Wurzeln bzw. der intellektuellen Ursprünge von Forschungsfeldern geeignet.

Eine solche Analyse muss immer im fachlichen Kontext und mit dem notwendigen Fachwissen vorgenommen werden. Die RPYS-Methode kann einen Wissenschaftshistoriker niemals ersetzen, aber sie kann seine Arbeit wesentlich unterstützen. Die Anwendung dieser Methode liefert eine Liste aller zitierten Arbeiten, aus denen man dann die meist zitierten Referenzen ermitteln kann. Diese verweisen im Allgemeinen auf diejenigen Publikationen, die im Rahmen der historischen Aufarbeitung eines Forschungsfeldes potentiell von Bedeutung sind. Man bekommt quantitative Daten, die gewissermaßen das Ergebnis einer „Abstimmung“ der Gesamtheit der publizierenden Fachkollegen über deren Zitierungen darstellt. Über die Auswahl der Referenzen in ihren Publikationen dokumentieren die Fachkollegen, welche Arbeiten in welchem Jahr aus ihrer Sicht besonders wichtig waren. Während der Historiker seine Verweise in Form von Referenzen immer subjektiv auswählt, liefert die Gesamtheit der Fachkollegen ein objektiveres Bild.

Die bisherigen Arbeiten zu Anwendungen der RPYSMethode konnten die wesentlichen Publikationen der jeweiligen Forschungsfelder erfolgreich identifizieren. Einige Beispiele sind die Entstehung der Graphenforschung und die Entwicklung der Solarzellen (Photovoltaik) (Marx et al. 2014), die Entwicklung des Global Positioning System (GPS) (Comins \& Hussey 2015), die Geschichte des HiggsBosons (Barth et al. 2014), die Entstehung der Legende von den Darwin-Finken (Marx \& Bornmann 2014), die Entwicklung der Bibliometrie (Leydesdorff et al. 2014) oder die Geschichte der Klimaforschung (Marx et al. 2016). Einen Überblick zu den wesentlichen Anwendungen von Referenz-basierten Daten findet man in Marx \& Bornmann (2016).

Der Ausgangspunkt ist immer ein geeigneter Publikationsset, der möglichst viele relevante Arbeiten zu dem betreffenden Forschungsfeld erfasst und über diverse Methoden der Datenbanksuche zusammengestellt wird. Aus diesem Publikationsset werden dann alle Referenzen extrahiert, die in den einzelnen Publikationen zitiert wurden, und hinsichtlich ihrer Erscheinungsjahre analysiert. $\mathrm{Zu}$ diesem Zweck wurde das Programm Cited References Explorer (CRExplorer) entwickelt (Thor et al. 2016), das für jedermann frei zugänglich ist und die Auswertung der Rechercheergebnisse zu einer RPYS-Analyse wesentlich erleichtert. Da die Referenzen zumeist in vielen Varianten erscheinen, die von den Datenbank-Herstellern kaum standardisiert sind, ist für die weitere Auswertung eine Erkennung und Zusammenfassung der zusammengehörigen Referenzen notwendig. Dazu wurden für den CRExplorer geeignete Algorithmen entwickelt und auch die Möglichkeit der manuellen Bearbeitung vorgesehen. Dabei können auch fehlerhafte (verschriebene) Zitierungen berücksichtigt werden, die im Falle historischer Arbeiten wegen der langen Laufzeit häufiger „mutieren“ und deshalb besonders zahlreich sind (Marx 2011).

In dieser Arbeit stellen wir die RPYS-Methode anhand von zwei ausgewählten Beispielen vor, die jedermann mit Zugang zu WoS leicht nachvollziehen kann. Es handelt sich um das Forschungsfeld der Photovoltaik als Basis der Solarzellen-Technologie und den Treibhauseffekt als zentrales Thema der Klimaforschung vor dem Hintergrund der globalen Erwärmung.

Als Datenbasis können alle bibliographischen Datenbanken herangezogen werden, soweit sie auch die zitierten Referenzen der erfassten Publikationen enthalten. Im ersten Schritt werden die relevanten Arbeiten des zu untersuchenden Forschungsfeldes zusammengestellt und samt den darin zitierten Referenzen heruntergeladen. Im zweiten Schritt werden die Zitierhäufigkeiten gegen die Publikationsjahre der zitierten Referenzen aufgetragen. Die RPYS-Methode besteht also aus zwei Schritten: (1) einer Datenbanksuche nach der Literatur des jeweiligen Forschungsfeldes und der Erzeugung der RPYS-Rohdaten sowie (2) der Generierung der finalen Ergebnisse zur vertieften Analyse, z. B. mittels des CRExplorer.

Zunächst wird ein Forschungsfeld ausgewählt (hier: Photovoltaik sowie Treibhauseffekt) und durch eine geeignete Suchformulierung (Query) beschrieben. Die Formulierung der Query und die anschließende Datenbanksuche gehören $\mathrm{zu}$ den Alltagsaufgaben eines jeden Informationsspezialisten. Manche Themen lassen sich relativ einfach mit Hilfe von wenigen Schlagwörtern beschreiben, andere erfordern eine subtilere Vorgehensweise. Da die RPYS-Methode auf einer statistischen Analyse beruht, muss das Ergebnis der Literaturrecherche nicht unbedingt alle relevanten Publikationen des Forschungsfeldes (und nur diese) umfassen. Wenn einige Publikationen fehlen oder auch falsche Publikationen erfasst wer- 


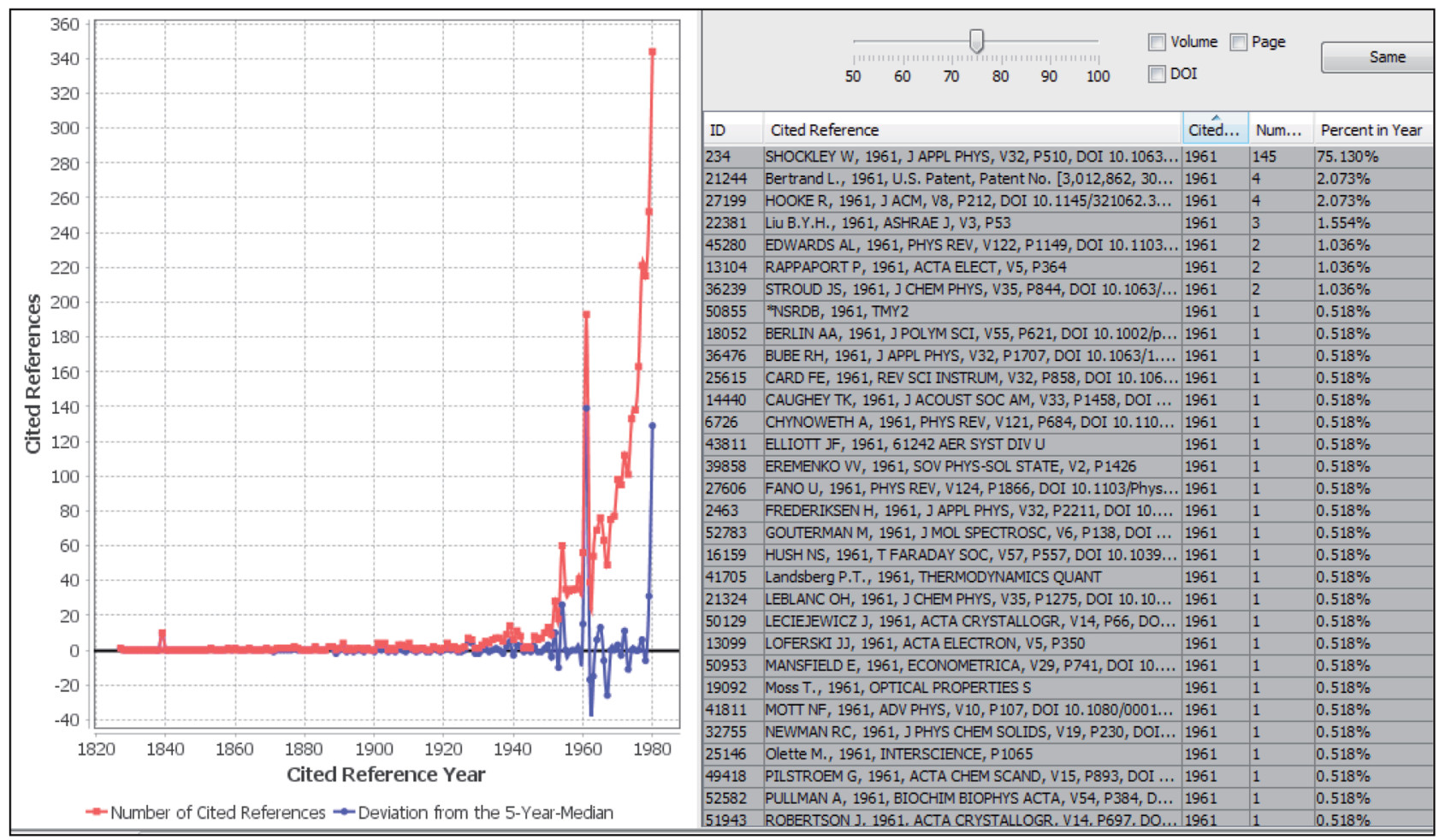

Abbildung 2: Screenshot des CRExplorer. Hier sieht man links das Spektrum zum Forschungsfeld Photovoltaik und in der Tabelle rechts daneben die Cited References für das Jahr 1961 in absteigender Reihenfolge ihrer Häufigkeit.

den, dann wird dies das Ergebnis im Allgemeinen nicht signifikant beeinflussen. In unseren Beispielen beschränken wir uns auf einfache Queries mit Suchen im Titelfeld von WoS, damit die Leserschaft die Literatursuche leichter nachvollziehen kann.

Im zweiten Schritt müssen die RPYS-Rohdaten für die Analyse prozessiert werden. Dazu werden die zitierten Referenzen aus dem Publikationsset extrahiert. Man erhält dann einen zweiten Antwortsatz, der aus allen Referenzen aller aufgefundenen Publikationen zu dem ausgewählten Forschungsfeld besteht. Man hat jetzt alle Rückwärtszitierungen zu dem Forschungsfeld vorliegen und dies bedeutet einen Wechsel der Perspektive gegenüber der Bibliometrie im Allgemeinen. Der RPYS-Antwortsatz muss exportiert werden und kann dann direkt in MS Excel aufgearbeitet werden. Im Prinzip muss man den Antwortsatz in eine Excel-Tabelle überführen und dann nach dem Reference Publication Year (RPY) und der Häufigkeit der Referenzen sortieren. Diese Vorgehensweise stößt aber auf die Schwierigkeit, dass es sich bei dem RPYS-Antwortsatz um Rohdaten handelt. Aufgrund von unterschiedlichen Schreibweisen, z.B. der Zeitschriftentitel, und von Verschreibungen ist der Antwortsatz noch relativ fehlerbehaftet. Wenn man die direkte Aufarbeitung in MS Excel wählt, muss man daher sehr viel Arbeit in die Korrektur der Daten investieren.
Um die Schreibfehler zu minimieren und die Zeitschriftentitel zu standardisieren, werden stattdessen die Rohdaten mit dem CRExplorer prozessiert (Thor et al 2016). Auf der Webseite findet man eine ausführliche Dokumentation sowie ausgewählte Beispiele (http://www.crexplorer.net/). Der CRExplorer bietet darüber hinaus den entscheidenden Vorteil, dass Grafik und Referenzlisten aufeinander abgestimmt analysiert und bearbeitet werden können. Als Ergebnis der Prozessierung erhält man die korrigierten Daten zur genaueren Analyse sowie das grafische Spektrum. Es sollte noch erwähnt werden, dass manchmal noch eine finale manuelle Überarbeitung der Daten erforderlich ist, weil die Software nicht alle unterschiedlichen Schreibweisen, Verschreibungen und Zitierweisen erkennen kann. In der Abbildung 2 ist ein Screenshot des CRExplorer zur Analyse der Photovoltaik zu sehen: Die Grafik zeigt das Spektrum und in der Tabelle sind die Cited References für das Jahr 1961 in nach Häufigkeit absteigender Reihenfolge zu sehen. Man erkennt, dass eine einzige Arbeit das Jahr 1961 dominiert (siehe dazu das erste Beispiel einer RPYS-Analyse unten).

Zur Analyse der Geschichte des Treibhauseffektes (siehe dazu das zweite Beispiel weiter unten) erweitern wir die RPYS-Methode durch die Verwendung von Ko-Zitierungen einer ausgewählten Arbeit als Publikationsbasis für die Analyse. Es werden alle ko-zitierten Referenzen einer 


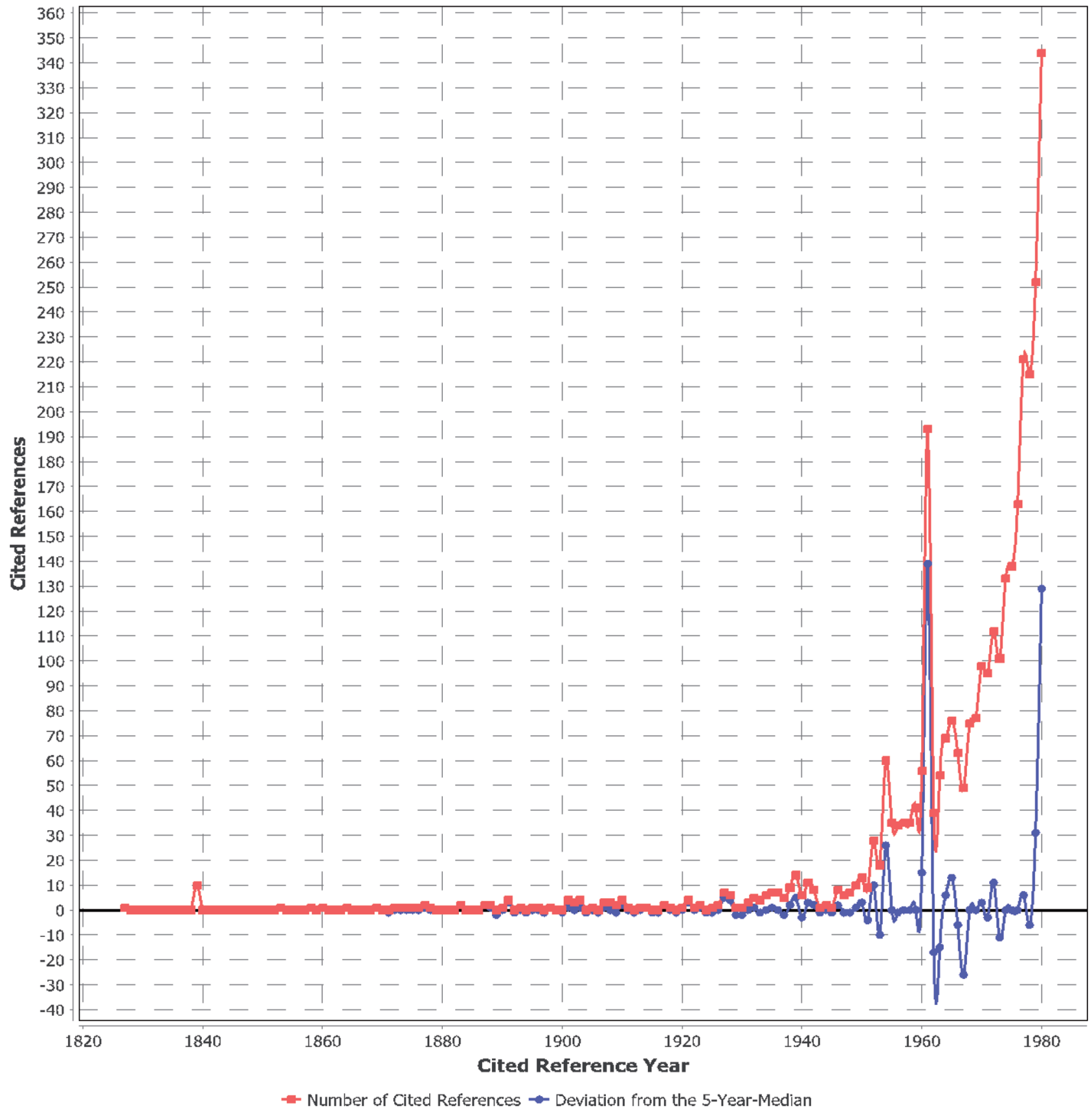

Abbildung 3: Zeitliche Entwicklung (1800 - 1980) der zitierten Literatur zum Thema „photovoltaics“ (Quelle: WoS, Oktober 2016).

bahnbrechenden Arbeit herangezogen, d.h. alle Referenzen in allen Arbeiten, die eine bestimmte Arbeit zitieren. Die ausgewählte Arbeit dient jeweils als Marker für das entsprechende Thema, indem deren Zitierungsumgebung in Form der ko-zitierenden Referenzen selektiert und mittels RPYS analysiert wird. Die Marker-Arbeit sollte eine Publikation sein, deren Berücksichtigung als Referenz man in wissenschaftshistorischen Abhandlungen voraussetzen kann. Auf diese Weise wird auf die historisch relevanten Publikationen und deren Referenzen fokussiert, die im gesamten Publikationsset zu einem Forschungsfeld (siehe die erste Analyse) lediglich einen geringen Anteil einnehmen und eher ein Zitat-Rauschen darstellen.

\section{Die Ursprünge der Photovoltaik}

Unter Photovoltaik versteht man „die direkte Elektrizitätsgewinnung aus eingestrahlter Sonnenenergie mit Hilfe von dotierten Halbleitermaterialien“(Roempp 2016). Eine prak- 
Tabelle 1: Die Publikationen zur Photovoltaik mit der höchsten Anzahl von Zitierungen im jeweiligen Jahrgang. RPY bezeichnet das Reference Publication Year und NCR ist die Anzahl der Zitierungen. In Klammern daneben der Prozentanteil im jeweiligen Jahrgang (Number of Cited References).

\begin{tabular}{|c|c|c|}
\hline Nr. & RPY Zitierte Referenz & NCR \\
\hline CR1 & $\begin{array}{l}1839 \text { Becquerel, A.E. Mémoire sur les effets électriques produits sous l'influence des rayons solaire. Comptes Rendus de } \\
\text { l'Académie des Sciences 9, 561-567. }\end{array}$ & $10(100 \%)$ \\
\hline CR2 & $\begin{array}{l}1839 \text { Becquerel, A.E. (1839). Recherches sur les effets de la radiation chimique de la lumière solaire au moyen des } \\
\text { courants électriques. Comptes Rendus Hebdomadaires des Séances de l'Académie des Sciences 9, 145-149. }\end{array}$ & \\
\hline CR3 & $\begin{array}{l}1952 \text { Shockley, W.; Read, W.T. Statistics of the recombinations of holes and electrons. Physical Review 87(5), 835-842. } \\
\text { DOI: 10.1103/PhysRev.87.835. }\end{array}$ & $11(39 \%)$ \\
\hline CR4 & $\begin{array}{l}1954 \text { Chapin, D.M.; Fuller, C.S.; Pearson, G.L. A New Silicon p-n Junction Photocell for Converting Solar Radiation into } \\
\text { Electrical Power. Journal of Applied Physics 25(5), 676-677, DOI: 10.1063/1.1721711 }\end{array}$ & $19(32 \%)$ \\
\hline CR5 & $\begin{array}{l}1961 \text { Shockley, W.; Queisser, H.J. Detailed balance limit of efficiency of } p \text {-n junction solar cells. Journal of Applied Physics } \\
\text { 32(3), 510-519. DOI: } 10.1063 / 1.1736034 .\end{array}$ & $145(75 \%)$ \\
\hline
\end{tabular}

tische Anwendung findet man in Solarzellen, die inzwischen auch auf den Dächern vieler Privathäuser installiert sind. Für die vorliegende Arbeit haben wir eine einfache Query gewählt, um das Prinzip der RPYS-Analyse zu illustrieren. Mit der Suche nach dem Wort „photovoltaics“ im Titelfeld des WoS ${ }^{2}$ erhält man 3.296 Publikationen (Oktober 2016), die man in mehreren Schritten in Paketen von jeweils 500 Dokumenten aus dem WoS herunterladen kann. Ausgewählt wurden (wie bei bibliometrischen Analysen üblich) die Dokumenttypen „articles“, „proceeding papers“ und „reviews“. Die WoS-Dateien wurden dann in den CRExplorer importiert und hinsichtlich der im Publikationsset zitierten Referenzen analysiert. In diesem Falle handelt es sich um insgesamt 52.950 zitierte Referenzen. Der CRExplorer liefert eine grafische Darstellung der Häufigkeit der Referenzen (cited references) nach den Publikationsjahren (cited reference year). Zusätzlich bekommt man eine Tabelle aller zitierten Referenzen und kann weitere Informationen einblenden. Mit Hilfe diverser Parameter kann man den Zeitraum so einschränken, dass man ein aussagekräftiges Spektrum erhält. Für die Photovoltaik haben wir den Zeitraum 1800 bis 1980 gewählt (s. Abb. 3); in diesen Zeitraum fallen 2.920 zitierte Referenzen.

Man erkennt deutlich vier Peaks, und zwar in den Jahren 1839, 1952, 1954 und 1961. Danach geht das Spektrum in eine Art Kontinuum über. Dominiert wird das Photovoltaik-Spektrum von einem einzigen Peak im Jahr 1961, der auf die berühmte Arbeit von Shockley und Queis-

2 TITLE: (photovoltaics), refined by: DOCUMENT TYPES: (ARTICLE OR PROCEEDINGS PAPER OR MEETING ABSTRACT), Indexes=SCIEXPANDED, SSCI, A\&HCI, CPCI-S, CPCI-SSH, BKCI-S, BKCI-SSH, ESCI, CCR-EXPANDED, IC Timespan=All years. ser zum maximal möglichen Wirkungsgrad von Solarzellen verweist. In der Tabelle 1 sind die vier hochzitierten Arbeiten für die o.g. Jahre zusammengestellt.

Hinweis: Da die NCR-Werte dieser und der folgenden Tabellen die Zitierungen von Arbeiten aus unterschiedlichen Publikations- und Zitierkulturen beinhalten, sind sie ohne entsprechende Normierung nicht miteinander vergleichbar und deshalb auch nicht als Ranking aufzufassen.

Im Jahr 1839 publizierte Becquerel (1820-1891) zwei Arbeiten, in denen er erstmalig den Bau einer Solarzelle beschrieb (CR1, CR2: 1839). Danach gab es über 100 Jahre lang keine wesentlichen Beiträge und das Spektrum zeigt daher einen flachen Verlauf nahe Null. Erst im Jahr 1952 publizierten Shockley (1910-1989) und Read ihre Arbeit über die Rekombination eines Elektrons mit einem Lochzustand unter Abstrahlung eines Photons (CR3: 1952). Im Jahr 1954 wurde dann die erste moderne Solarzelle von Daryl Chapin (1906-1995) zusammen mit Calvin Fuller und Gerald Pearson in den Bell Laboratories entwickelt und der Öffentlichkeit in einem großen Medienereignis vorgestellt. Als Technik verwendeten sie die Diffusion von Bor (p-Typ) in Silizium (n-Typ) (CR4: 1954).

Der dominante Peak im Jahr 1961 basiert auf der Arbeit von Shockley \& Queisser („Detailed balance limit of efficiency of $p$ - $n$ junction solar cells") (CR5: 1961). William Bradford Shockley (1910-1989) hatte über Halbleiter geforscht und im Jahr 1956 zusammen mit John Bardeen und Walter Houser Brattain für die Forschungen zur Halbleiterphysik und die Entdeckung des Transistoreffektes den Nobelpreis für Physik erhalten. In der Arbeit von Shockley \& Queisser (1961) wird die sogenannte Shockley-QueisserGrenze berechnet, die den maximal möglichen Wirkungsgrad von Solarzellen darstellt. Mit 145 Zitierungen (75 \%) innerhalb unseres ausgewählten Publikationssets (die Gesamtzahl der Zitierungen über alle Fachgebiete ist wesent- 


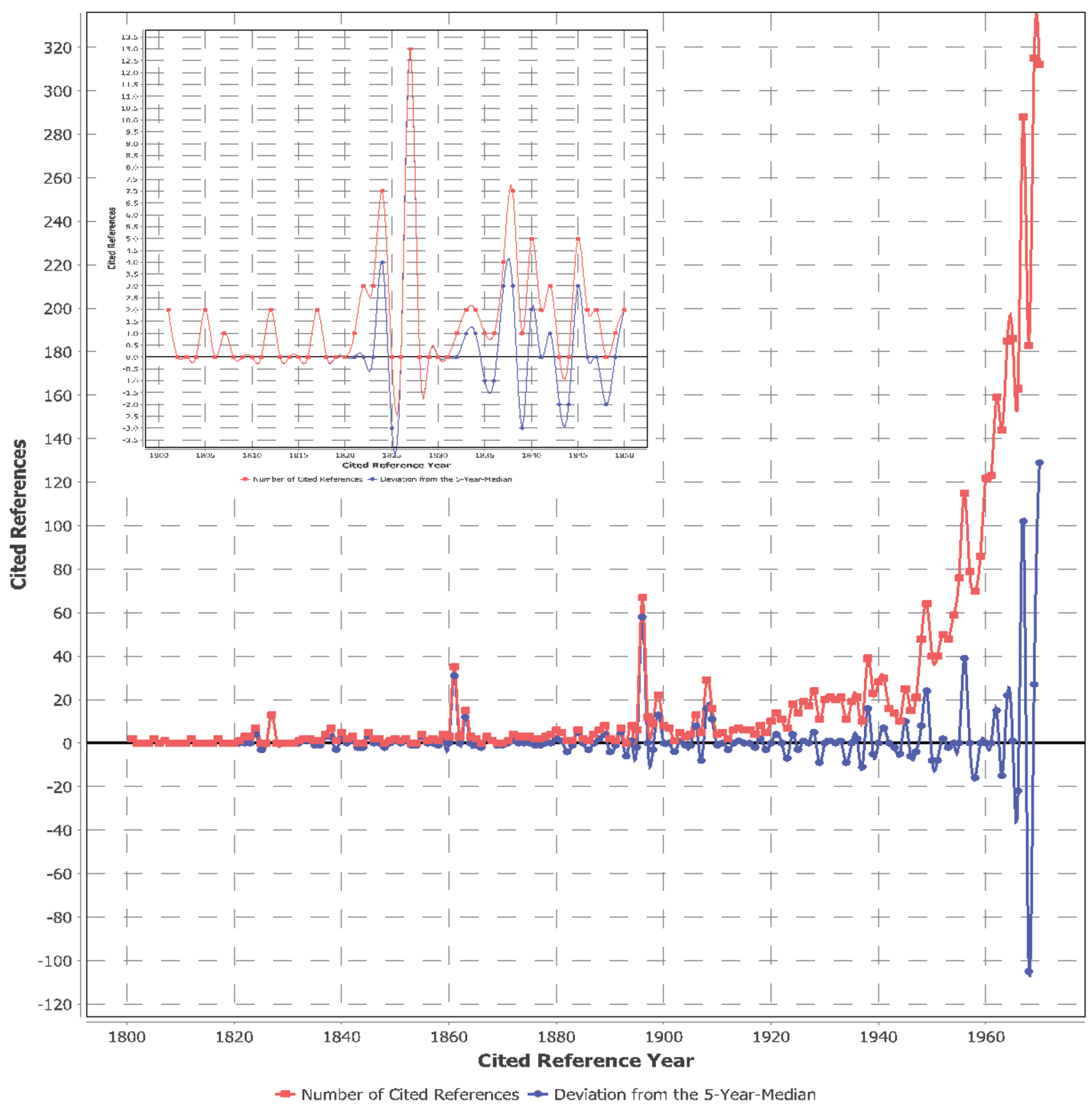

Abbildung 4: Zeitliche Entwicklung (1800 bis 1900) der zitierten Literatur zum Thema „greenhouse effect“. Zur besseren Identifikation der Peaks im Zeitabschnitt von 1800 bis 1850 wurde dieser Bereich vergrößert (oben links) (Quelle: WoS, Oktober 2016).

lich höher) ist dies die markanteste Arbeit in unserer Untersuchung zur Photovoltaik.

Eine ausführliche Untersuchung des Forschungsfeldes der Photovoltaik findet man in der Publikation von Marx et al. (2014). Auf Basis einer aufwändigeren Query zur Erfassung der Publikationen über Solarzellen und Photovoltaik wurden in der dort vorgestellten Analyse insgesamt 62.412 Publikationen gefunden und daraus 1.375.860 zitierte Referenzen extrahiert.

\section{Die Entdeckung des Treibhauseffektes}

„Allgemein veranschaulicht der Begriff Treibhauseffekt die Analogie der Effekte, welche zur Erwärmung der bodennahen Luftschicht der Erde einerseits und der Luft in einem Glashaus (Treibhaus) anderseits führen ... Wissenschaftliche Grundlage der Betrachtungen zum atmosphärischen Treibhauseffekt ist die Strahlungsbilanz, in welcher die Dif- 
Tabelle 2: Die Publikationen zum Treibhauseffekt mit den höchsten relativen Zitierungen im jeweiligen Jahrgang. RPY bezeichnet das Reference Publication Year und NCR ist die Anzahl der Zitierungen. In Klammern daneben der Prozentanteil im jeweiligen Jahrgang (Number of Cited References).

\begin{tabular}{|c|c|c|}
\hline Nr. & RPY Referenz & NCR \\
\hline CR1 & $\begin{array}{l}1824 \text { Jean Baptiste Joseph Fourier: Remarques Générales sur les Température du Globe Terrestre et des Espaces } \\
\text { Planétaires. Annales de Chimie et de Physique 27, 136-167. }\end{array}$ & $7(100 \%)$ \\
\hline CR2 & $\begin{array}{l}1827 \text { Jean Baptiste Joseph Fourier: Mémoire sur les températures du globe terrestre et des espaces planétaires. } \\
\text { Mémoires de l'Académie Royale des Sciences 7, 569-604. }\end{array}$ & $12(92 \%)$ \\
\hline CR3 & $\begin{array}{l}1861 \text { John Tyndall: On the absorption and radiation of heat by gasses and vapours, and on the physical connection of } \\
\text { radiation, absorption, and conduction. Philosophical Magazine, Series 4, 22: 273-285. }\end{array}$ & $34(97 \%)$ \\
\hline CR4 & 1863 John Tyndall: On Radiation through the Earth's Atmosphere. Philosophical Magazine, Series 4, 25: 200-206. & $11(73 \%)$ \\
\hline CR5 & $\begin{array}{l}1896 \text { Svante Arrhenius: On the influence of carbonic acid in the air upon the temperature of the ground. Philosophical } \\
\text { Magazine and Journal of Science Series 5(41), 237-276. }\end{array}$ & $62(93 \%)$ \\
\hline CR6 & 1897 Thomas Chrowder Chamberlin: A group of hypotheses bearing on & $7(58 \%)$ \\
\hline CR7 & $\begin{array}{l}1898 \text { Thomas Chrowder Chamberlin: The influence of great epochs of limestone formation upon the constitution of the } \\
\text { atmosphere. Journal of Geology 6, 609-621. }\end{array}$ & $6(67 \%)$ \\
\hline CR8 & $\begin{array}{l}1899 \text { Thomas Chrowder Chamberlin: An attempt to frame a working hypothesis on the cause of glacial periods on an } \\
\text { atmospheric basis. Journal of Geology } 7,545-584,667-685,751-787 \text {. }\end{array}$ & $17(77 \%)$ \\
\hline CR9 & $\begin{array}{l}1908 \text { Svante Arrhenius: Worlds in the making: the evolution of the universe. Harper \& Brothers Publishers, New York \& } \\
\text { London (264 pages). }\end{array}$ & $9(31 \%)$ \\
\hline CR10 & $\begin{array}{l}1924 \text { Jean Baptiste Joseph Fourier: Remarques Générales sur les Températures du Globe Terrestre et des Espaces } \\
\text { Planétaires. Annales de Chimie et de Physique 27, 136-167. }\end{array}$ & $9(50 \%)$ \\
\hline CR11 & $\begin{array}{l}1938 \text { Guy Stewart Callendar: The artificial production of carbon dioxide and its influence on temperature. Quarterly } \\
\text { Journal of the Royal Meteorological Society } 64,223-237 .\end{array}$ & $19(49 \%)$ \\
\hline CR12 & 1949 Guy Stewart Callendar: Can carbon dioxide influence climate? Weather 4, 310-314. & $6(9 \%)$ \\
\hline CR13 & 1956 Gilbert Norman Plass: The carbon dioxide theory of climatic change. Tellus 8, 140-154. & $12(10 \%)$ \\
\hline
\end{tabular}

ferenz aus von der Sonne eingestrahlter (importierter) Energie und von der Erdoberfläche zurückgestrahlter (exportierter) Energie erfasst wird. “(Römpp 2016)

Zur Zusammenstellung eines Publikationssets zum Treibhauseffekt suchen wir in WoS (Stand: Oktober 2016) mit dem Term „greenhouse effect“ im Topicfeld (d.h. im Titel-, Abstract- und Schlagwortfeld), und es werden wiederum nur die Dokumenttypen „articles“, „proceeding papers“ und „reviews“ berücksichtigt. ${ }^{3}$ Die Erweiterung der Suchfelder über das Titelfeld hinaus auch auf die Abstracts und Schlagworte war notwendig, um eine ausreichende Datengrundlage vergleichbar zum ersten Beispiel herzustellen. Als Ergebnis erhält man 3.244 Publikationen, aus denen 79.032 zitierte Referenzen extrahiert werden. In der Abbildung 4 findet man das Spektrum für den Zeitraum 1800 bis 1970. Um die Möglichkeiten einer vertieften Analyse durch Einzoomen im Spektrum zu demonstrieren und die Ursprünge in Form der am weitesten zurückliegenden historischen Arbeiten von Joseph Fourier besser erkennen

3 TOPIC: ("greenhouse effect"), refined by: DOCUMENT TYPES: (ARTICLE OR PROCEEDINGS PAPER OR REVIEW), Indexes=SCI-EXPANDED, SSCI, A\&HCI, CPCI-S, CPCI-SSH, BKCI-S, BKCI-SSH, ESCI, CCR-EXPANDED, IC Timespan=All years. zu können, wurde der Bereich von 1800 bis 1850 vergrößert und oben links in die Abbildung eingefügt.

In der Abbildung 4 kann man die wichtigsten Peaks für den Zeitabschnitt 1800 bis 1970 leicht erkennen. Man findet Peaks für die Jahre 1824, 1827, 1861, 1863, 1896, 1897, 1898, 1899, 1908, 1924, 1938, 1949 und 1956. Die Peaks 1897 und 1898 sowie die dazugehörigen Arbeiten können allerdings nur mit Hilfe der ausführlichen Tabelle aus dem CRExplorer identifiziert werden. Eine Zusammenfassung der historisch relevanten Arbeiten findet man in Tabelle 2.

Als Ergebnis der RPYS-Analyse findet man die wichtigsten Arbeiten zum Treibhauseffekt, zusammen mit der Anzahl der Zitierungen im jeweiligen Publikationsjahr. Der französische Mathematiker und Physiker Joseph Fourier (1768-1830) stellte fest, dass die Erde wärmer war, als sie nach seiner Abschätzung sein sollte (CR1: 1824, CR2: 1827). Er führte dies darauf zurück, dass die Erdatmosphäre zwar für das Sonnenlicht transparent ist, nicht aber für die durch Erwärmung des Erdbodens und der Atmosphäre erzeugte Infrarotstrahlung, und damit hatte er den (natürlichen) Treibhauseffekt entdeckt. Die Arbeit von 1924 (CR10) erscheint meistens mit dem falschen Erscheinungsjahr (korrekt: 1824); dies ist auf einen Fehler in der Datenbank zurückzuführen. Der Beitrag von Fourier zum Treib- 
hauseffekt wird in vielen, aber bei weitem nicht in allen wissenschaftshistorischen Arbeiten gewürdigt (siehe dazu z. B. Fleming, 1999).

Der irische Physiker John Tyndall (1820-1893) konnte als Erster die Wärmestrahlung von Treibhausgasen wie Kohlendioxid und Wasserdampf messen (CR3: 1861, CR4: 1863). Er vermutete, dass Änderungen in der Konzentration dieser Gase zu einer Klimaveränderung führen könnten. Die Experimente von Tyndall haben maßgeblichen Anteil an der Erforschung des Treibhauseffektes (Hulme 2009). Im Jahr 1896 berechnete der schwedische Chemiker Svante Arrhenius (1859-1927) die quantitativen Auswirkungen der Konzentration von Kohlendioxid in der Atmosphäre auf die Veränderung der Temperatur der Erdoberfläche (CR5: 1896). In dem Buch „Worlds in the making: the evolution of the universe “ (deutsch: „Das Werden der Welten“) (CR9: 1908) formulierte er seine Theorie in allgemeinverständlicher Form. Arrhenius konnte vorhersagen, dass die Verbrennung fossiler Brennstoffe ausreichend ist, um eine globale Erwärmung auszulösen. Seine Vorhersage zum globalen Temperaturanstieg durch eine Verdopplung der $\mathrm{CO}_{2}$ Konzentration liegt nahe an den Vorhersagen auf der Basis moderner Klimamodelle. Die Experten lehnten seine Hypothese jedoch zunächst ab, weil sie seine Berechnung als zu grobe Vereinfachung des Problems ansahen. Die Arbeiten des Geologen Thomas Crowder Chamberlin (1843-1928) (CR6: 1897, CR7: 1888, CR8: 1889) unterstützten die Theorie von Arrhenius, Guy Stewart Callendar (1898-1964) entwickelte die erste vollständige Theorie der Klimaveränderung (CR11: 1938, CR12: 1949) und Gilbert Norman Plass (1920-2004) berechnete die Transmission der Strahlung durch die Erdatmosphäre (CR13: 1956).

\section{Die Entdeckung des Treibhauseffektes, analysiert auf Basis einer Ko-Zitierungsanalyse}

Die Masse der Publikationen zu einem Forschungsfeld ist auf den aktuellen wissenschaftlichen Diskurs gerichtet und nicht auf die historischen Arbeiten. Da in der Regel nur ein vergleichsweise kleiner Anteil der Publikationen zu einem Forschungsfeld die historischen Arbeiten zitiert, kommt es zu einem Verdünnungseffekt. Um diesen Verdünnungseffekt hinsichtlich der historisch relevanten Referenzen zu vermeiden, kann man der RPYS-Analyse ein Publikationsset zugrunde legen, das lediglich diejenigen Arbeiten umfasst, die mindestens eine (oder auch mehrere) prominente und historisch besonders wichtige Arbeit zitieren. Man kann davon ausgehen, dass Publikationen, die eine solche Marker-Arbeit zitieren, auch die potentiellen Zitierer der anderen historisch relevanten Arbeiten sind. Damit untersucht man statt aller in Frage kommenden Referenzen im jeweiligen Publikationsset gezielt die Zitierumgebung einer Marker-Arbeit, d.h. alle Referenzen, welch mit der betreffenden Arbeit gemeinsam zitiert (kozitiert) wurden. Hier bietet sich die Heranziehung einer bibliometrischen Methode an, die in anderem Zusammenhang vielfache Anwendung gefunden hat: die Methode der Ko-Zitierungsanalyse. Diese Methode macht sich zu Nutze, dass gleichzeitig von einer Publikation zitierte (ko-zitierte) Arbeiten inhaltlich miteinander verwandt sind und untersucht z.B. die Verwandtschaftsbeziehung von Autoren oder Zeitschriften. Im Folgenden wird die Methode jedoch zur gezielten Zusammenstellung eines Publikationssets verwendet, um auf die historisch relevanten Publikationen $\mathrm{zu}$ fokussieren. Die RPYS-Analyse der so aufgefundenen Referenzen erfolgt dann ganz analog zum vorigen Beispiel. Das Konzept zur Kombination der Ko-Zitierungsanalyse mit der RPYS-Analyse wurde von Marx et al. (2016) entwickelt und auf das Gebiet der Klimaforschung angewandt.

Alle Publikationen, die z.B. die Arbeiten von Svante Arrhenius von 1896 und 1908 zum Einfluss des atmosphärischen Kohlendioxids auf die Oberflächentemperatur der Erde zitieren, werden vermutlich auch die anderen wichtigen (frühen) Arbeiten zum Treibhauseffekt zitieren. Im Vergleich zur Gesamtheit der Fachliteratur der Klimaforschung schneidet man damit denjenigen Anteil heraus, der sich potentiell mit der historischen Seite dieses Forschungsthemas beschäftigt. Man darf erwarten, dass man über diese Methode auch eventuelle Vorläuferarbeiten ermitteln kann. Solche Arbeiten werden nicht immer ihrer Bedeutung entsprechend zitiert, weil man sich oft mit der Zitierung einer einzigen (zumeist besonders prominenten) Arbeit zufrieden gibt. Auch die Wissenschaft ist hier manchmal erstaunlich subjektiv, indem sie sich auf einen ausgewählten „Helden der Forschung“ geeinigt hat und somit die Anfänge eines Forschungsfeldes verkürzt und damit verzerrt darstellt.

Zur Untersuchung der Entdeckung des Treibhauseffektes wurde die Arbeit von Arrhenius von 1896 (CR5) als Marker-Arbeit im Cited Reference Search Modus des WoS aufgesucht und alle Arbeiten ermittelt, die diese MarkerArbeit zitieren. Dabei wurde auch seine Publikation von 1908 (CR9) ${ }^{4}$ berücksichtigt. Denn beide Veröffentlichun-

4 CITED AUTHOR: (Arrhenius S*) AND CITED YEAR: (1896 OR 1908), Indexes=SCI-EXPANDED, SSCI, A\&HCI, CPCI-S, CPCI-SSH, BKCI-S, BKCI-SSH, ESCI, CCR-EXPANDED, IC Timespan=All years. 


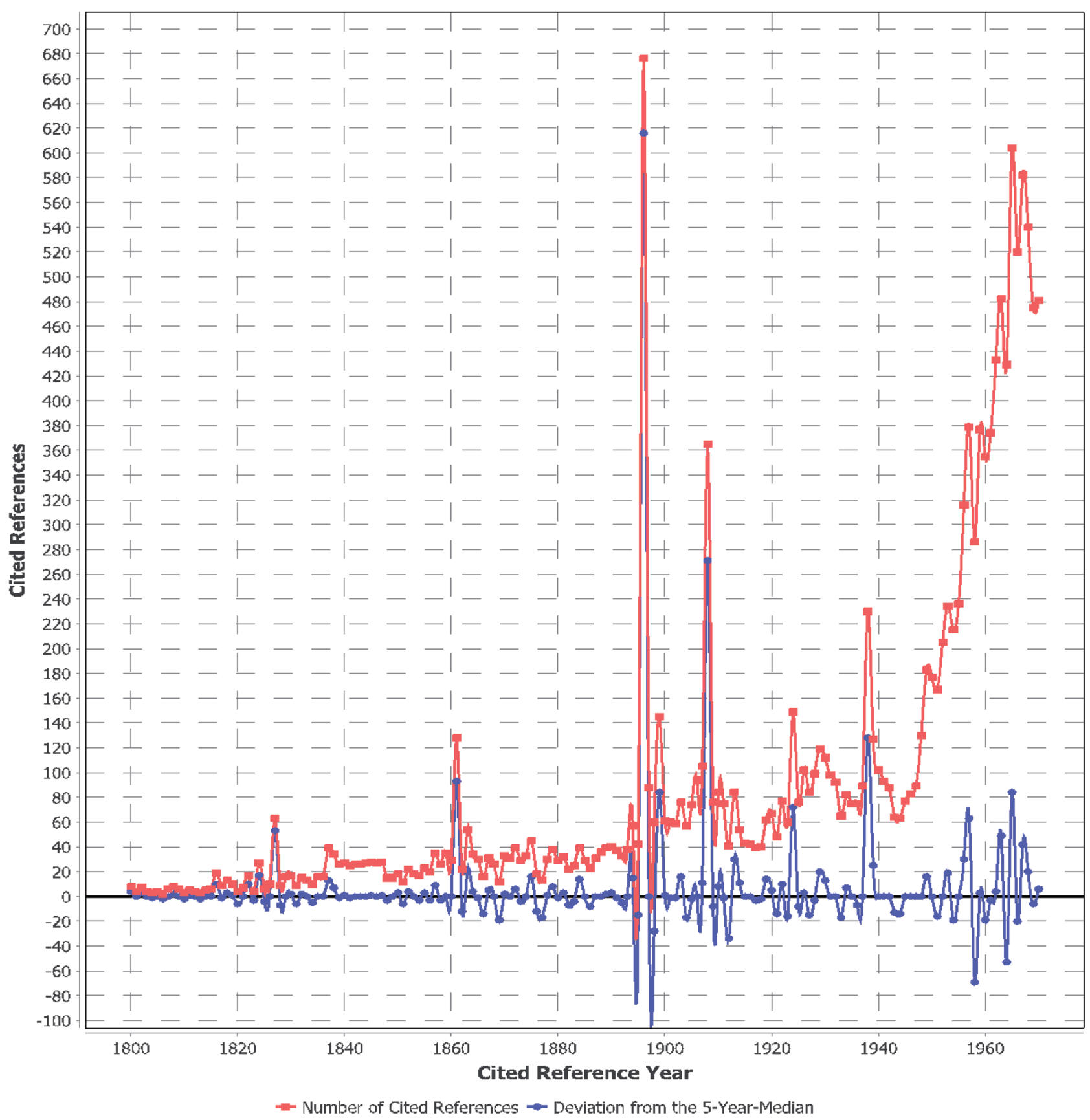

Abbildung 5: Zeitliche Entwicklung (1800 bis 1970) der zu den Arrhenius-Arbeiten von 1896 und 1908 ko-zitierten Literatur (Quelle: WoS, Oktober 2016).

gen können inhaltlich als Einheit betrachtet werden, weil die von 1908 eine populärwissenschaftliche Version der Arbeit von 1896 darstellt. Bei der Zusammenstellung des Publikationssets müssen auch fehlerhafte Zitierungen berücksichtigt werden, weil deren Anteil im Falle historischer Arbeiten nicht vernachlässigbar ist (Marx 2011). Die zitierenden Arbeiten der ausgewählten Marker-Arbeiten wurden im WoS samt Referenzen heruntergeladen und anschließend in den CRExplorer hochgeladen. Im Falle der beiden Arrhenius-Arbeiten waren dies 812 Publikationen (citing papers) mit insgesamt 63.108 Referenzen (cited references). Nach der Begrenzung auf den Zeitraum 1800 bis 1970 verblieben 12.394 Referenzen, deren Verteilung über die Erscheinungsjahre in Abbildung 5 graphisch dargestellt wird.

Das RPYS-Spektrum zeigt die Zitierungsumgebung der Arrhenius-Arbeiten von 1896 und 1908. Diejenigen Publikationen, die eine der beiden Arbeiten von Svante 
Tabelle 3: Die wichtigsten Publikationen zum Treibhauseffekt auf der Grundlage der Zitierungsumgebung der beiden Arbeiten von Arrhenius von 1896 und 1908. Die Nummerierung bezeichnet die ko-zitierten Arbeiten, RPY das Reference Publication Year und NCOR ist die Anzahl der Ko-Zitierungen. In Klammern daneben der Prozentanteil im jeweiligen Jahrgang.

\begin{tabular}{|c|c|c|}
\hline Nr. & RPY Referenz & NCOR \\
\hline COR1 & $\begin{array}{l}1824 \text { Jean Baptiste Joseph Fourier: Remarques Générales sur les Température du Globe Terrestre et des Espaces } \\
\text { Planétaires. Annales de Chimie et de Physique 27,136-167. }\end{array}$ & $15(56 \%)$ \\
\hline COR2 & $\begin{array}{l}1827 \text { Jean Baptiste Joseph Fourier: Mémoire sur les températures du globe terrestre et des espaces planétaires. } \\
\text { Mémoires de l'Académie Royale des Sciences 7, 569-604. }\end{array}$ & $46(73 \%)$ \\
\hline COR3 & $\begin{array}{l}1861 \text { John Tyndall: On the absorption and radiation of heat by gasses and vapours, and on the physical connection } \\
\text { of radiation, absorption, and conduction. Philosophical Magazine, Series 4, 22: 273-285. } \\
1863 \text { John Tyndall: On Radiation through the Earth's Atmosphere. Philosophical Magazine, Series 4, 25: 200-206. }\end{array}$ & $\begin{array}{r}104(81 \%) \\
30(56 \%)\end{array}$ \\
\hline COR5 & $\begin{array}{l}1896 \text { Svante Arrhenius: On the influence of carbonic acid in the air upon the temperature of the ground. } \\
\text { Philosophical Magazine and Journal of Science Series 5(41), 237-276. }\end{array}$ & $652(96 \%)$ \\
\hline COR6 & $\begin{array}{l}1897 \text { Thomas Chrowder Chamberlin: A group of hypotheses bearing on climatic changes. Journal of Geology } 5 \text {, } \\
\text { 653-683. }\end{array}$ & 46 \\
\hline COR7 & $\begin{array}{l}1898 \text { Thomas Chrowder Chamberlin: The influence of great epochs of limestone formation upon the constitution of } \\
\text { the atmosphere. Journal of Geology 6, 609-621. }\end{array}$ & $22(37 \%)$ \\
\hline COR8 & $\begin{array}{l}1899 \text { Thomas Chrowder Chamberlin: An attempt to frame a working hypothesis on the cause of glacial periods on an } \\
\text { atmospheric basis. Journal of Geology } 7,545-584,667-685,751-787 \text {. }\end{array}$ & $92(63 \%)$ \\
\hline COR9 & $\begin{array}{l}1908 \text { Svante Arrhenius: Worlds in the making: the evolution of the universe. Harper \& Brothers Publishers, New York } \\
\text { \& London (264 pages). }\end{array}$ & $220(60 \%)$ \\
\hline COR10 & $\begin{array}{l}1924 \text { Jean Baptiste Joseph Fourier: Remarques Générales sur les Températures du Globe Terrestre et des Espaces } \\
\text { Planétaires. Annales de Chimie et de Physique 27, 136-167. }\end{array}$ & $33(22 \%)$ \\
\hline COR11 & $\begin{array}{l}1938 \text { Guy Stewart Callendar: The artificial production of carbon dioxide and its influence on temperature. Quarterly } \\
\text { Journal of the Royal Meteorological Society } 64,223-237 \text {. }\end{array}$ & $114(50 \%)$ \\
\hline COR12 & 1949 Guy Stewart Callendar: Can carbon dioxide influence climate? Weather 4, 310-314. & $21(11 \%)$ \\
\hline COR13 & 1956 Gilbert Norman Plass: The carbon dioxide theory of climatic change. Tellus 8, 140-154. & $87(28 \%)$ \\
\hline
\end{tabular}

Arrhenius zitieren, sind auch die potentiellen Zitierer aller anderen in diesem Zusammenhang wichtigen (frühen) Arbeiten zum Treibhauseffekt. In allen Publikationen dieses Publikationssets muss mindestens eine der beiden Arrhenius-Arbeiten zitiert werden und deshalb sind die Peaks für die Jahre 1896 und 1908 besonders ausgeprägt. Aber auch die Peaks zu den anderen Arbeiten sind im Vergleich zu Abbildung 2 deutlicher ausgeprägt und besser differenziert. Das gilt insbesondere für die frühen Arbeiten von Joseph Fourier und John Tyndall. Ein breiter Peak folgt unmittelbar auf das Erscheinungsjahr der Arrhenius-Arbeit von 1896 und umfasst drei verhältnismäßig häufig zitierte Arbeiten aus den Jahren 1897 bis 1899. Weitere wichtige Peaks erkennt man für die Jahre 1908, 1924 und 1938. In der Tabelle 3 sind die wichtigsten Arbeiten und die Anzahl der Ko-Zitierungen zusammengefasst. Es handelt sich um dieselben Arbeiten wie in der Tabelle 2, allerdings haben die Ko-Zitierungen (NCOR) aus Tabelle 3 eine andere Bedeutung als die Zitierungen (NCR) aus Tabelle 2.

Mittels RPYS-Analyse basierend auf den Ko-Zitierungen einer sorgfältig ausgewählten Marker-Arbeit kann man also gezielt die historisch relevante Fachliteratur zu einem Forschungsfeld untersuchen und über den CRExplorer bis ins Detail verfolgen. In diesem Zusammenhang muss jedoch erwähnt werden, dass jedwede bibliometrische Analyse voraussetzt, dass eine Arbeit überhaupt zitiert und nicht von den Experten einfach vergessen wurde.

\section{Diskussion und Zusammenfassung}

Die RPYS-Analyse ist eine Methode zur quantitativen Erforschung der historischen Wurzeln von Forschungsfeldern. Anhand zweier aktueller Forschungsfelder haben wir gezeigt, wie RPYS-Analysen mit Hilfe einfacher Recherchen in der Datenbank WoS und einer anschließenden Auswertung mit dem CRExplorer durchgeführt werden können. Man findet zunächst alle zitierten Referenzen und kann dann über die Anzahl der Zitierungen die wesentlichen historischen Arbeiten identifizieren. Mit der „Abstimmung“ der publizierenden Experten hat man eine umfassende Datenbasis und ein quantitatives Maß für die 
Bedeutung der Beiträge im jeweiligen Publikationsjahr zur Verfügung. Die grafische Darstellung der Zitierhäufigkeiten (cited references) gegen die Publikationsjahre der Referenzen (cited reference year) ergibt eine Art Spektrum, aus dem man mit einem Blick die markantesten Referenzjahrgänge in Form von Peaks identifizieren kann. Ursache dieser relativ hohen Zitierhäufigkeiten sind meistens einzelne Publikationen, die einen wesentlichen Beitrag zu dem jeweiligen Forschungsfeld geleistet haben. Man kann also die geschichtliche Entwicklung eines Forschungsfeldes anhand der Ergebnisse der RPYS-Analyse „ablesen“ und rekonstruieren.

Im Falle der Photovoltaik werden die frühen Arbeiten von Becquerel (1839) erkennbar, aber auch die lange Zeit von einem ganzen Jahrhundert danach, in der keine nennenswerten Beiträge auftauchen. Im Gegensatz zum Thema Treibhauseffekt ist das Spektrum der RPYS-Analyse der Photovoltaik durch einen einzelnen markanten Peak im Jahr 1961 gekennzeichnet, der Arbeit von Shockley \& Queisser zur Bestimmung der Shockley-Queisser-Grenze, die den maximal möglichen Wirkungsgrad von Solarzellen aufzeigt.

Im Falle des Treibhauseffektes finden sich alle ab dem Anfang des neunzehnten Jahrhunderts erschienenen wesentlichen Arbeiten als ausgeprägte Peaks im Spektrum der RPYS-Analyse. Auch wenn die Zuweisung von Entdeckungen angesichts komplexer Zusammenhänge immer problematisch sein wird, so können die Beiträge von Joseph Fourier doch als historische Wurzel bzw. Entdeckung des Treibhauseffektes angesehen werden und gehen als solche auch eindeutig aus der RPYS-Analyse hervor. Sie werden gefolgt von den Arbeiten von Tyndall und Arrhenius und den Beiträgen von Callendar und Plass in der ersten Hälfte des zwanzigsten Jahrhunderts. Letzteren gebührt das Verdienst, trotz fast einhelliger Ablehnung durch die Fachkollegen, die Möglichkeit einer vom Menschen verursachten (anthropogenen) globalen Erwärmung ins Auge gefasst und wissenschaftlich untersucht zu haben. Erst die genauen Messungen der Kohlendioxid-Konzentration durch Charles David Keeling (19282005) haben dann ab 1960 den Nachweis erbracht, dass sich die Zusammensetzung der Erdatmosphäre durch den anthropogenen Ausstoß dieses Treibhausgases ändert und das Risiko einer globalen Erwärmung tatsächlich besteht.

In Ergänzung zu den RPYS-Analysen haben wir die Methode der Ko-Zitierungsanalyse verwendet, um die Zitierungsumgebung der beiden Arbeiten von Arrhenius (1896 und 1908) zum Treibhauseffekt von Kohlendioxid zu untersuchen. Hierbei haben wir uns von der Annahme leiten lassen, dass Publikationen, in denen bestimmte Marker-
Arbeiten zitiert werden, auch die anderen relevanten historischen Arbeiten als Referenzen enthalten. Die RPYSAnalyse aller ko-zitierten Referenzen liefert ein differenzierteres Bild von der Entwicklung des Treibhauseffektes.

Eine bibliometrische Methode wie die RPYS-Analyse liefert quantitative Ergebnisse zur Wissenschaftsgeschichte, basierend auf der Gesamtheit aller Zitierungen innerhalb der themenrelevanten Fachliteratur. Indem die bibliometrischen Daten auf der Gesamtheit der in der entsprechenden Fachliteratur referenzierten Arbeiten beruhen, können Unvollständigkeiten und Verzerrungen infolge subjektiver Auswahl durch die Historiker vermieden werden. Andererseits ist die Beurteilung der Ergebnisse und ihre Interpretation nicht sinnvoll ohne eben diese Experten. Die RPYS-Analyse kann diese bei ihrer Arbeit unterstützen aber nicht ersetzen.

Danksagung: Die Autoren bedanken sich bei Frau Gabriele Barth für die Übersetzungen des Abstracts.

\section{Literatur}

Barth, Andreas; Marx, Werner; Bornmann, Lutz; Mutz, Ruediger: On the origins and the historical roots of the Higgs boson research from a bibliometric perspective. In: The European Physical Journal - Plus (2014), 129(111), S. 1-13. http://dx.doi.org/do i:10.1140/epjp/i2014-14111-6.

Bornmann, Lutz; Marx, Werner: The wisdom of citing scientists. In: Journal of the American Society of Information Science and Technology (2014), 65(6), S. 1288-1292. http://dx.doi.org/do i:10.1002/asi.23100.

Comins, Jordan A.; Hussey, Thomas W.: Detecting seminal research contributions to the development and use of the global positioning system by reference publication year spectroscopy. In: Scientometrics (2015), 104(2), S. 575-580. http://dx.doi.org/doi: 10.1007/s11192-015-1598-2.

De Solla Price, Derek: Little Science, Big Science. New York, Columbia Press, 1963; dtsch., Suhrkamp, 1974.

Fleming, James R.: Joseph Fourier, the 'greenhouse effect', and the quest for a universal theory of terrestrial temperatures. In: Endeavor (1999), 23(2), S. 72-75.

Garfield, Eugene: Citation Indexing - Its Theory and Application in Science, Technology, and Humanities. John Wiley \& Sons, Inc. NY (1979).

Glänzel, Wolfgang: Bibliometrics as a research field: a course on theory and application of bibliometric indicators. Magyar Tudományos Akadémia, Kutatáss zervezési Intézet, Budapest (2003). http://yunus.hacettepe.edu.tr/ tonta/courses/spring2011/ bby704/bibliometrics-as-a-research-field-Bib_Module_KUL.pdf. Haunschild, Robin; Bornmann, Lutz; Marx, Werner: Climate Change Research in View of Bibliometrics. In: PLOS ONE (2016), 11(7): e0160393. http://dx.doi.org/10.1371/journal.pone.0160393.

Havemann, Frank: Einführung in die Bibliometrie. Gesellschaft für Wissenschaftsforschung Berlin (2009). http://www.wissen schaftsforschung.de/Havemann2009Bibliometrie.pdf. 
Hulme, Mike: On the origin of 'the greenhouse effect': John Tyndall's 1859 interrogation of nature. In: Weather (2009), 64(5), S. 121-123. http://dx.doi.org/doi:10.1002/wea.386.

Leydesdorff, Loet; Bornmann, Lutz; Marx, Wener; Milojevićc, Staša: Referenced Publication Years Spectroscopy applied to iMetrics. Scientometrics, Journal of Informetrics, and a relevant subset of JASIST. In: Journal of Informetrics (2014), 8(1), S. 162-174. http://dx.doi.org/doi:10.1016/j.joi.2013.11.006.

Marx, Werner: Special features of historical papers from the viewpoint of bibliometrics. In: Journal of the American Society for Information Science and Technology (2011), 62(3), S. 433-439. http://dx.doi.org/10.1002/asi.21479.

Marx, Werner; Haunschild, Robin; Thor, Andreas; Bornmann, Lutz: Which early works are cited most frequently in climate change research literature? In: Scientometrics (2016), im Druck. http:// dx.doi.org/doi:10.1007/s11192-016-2177-x.

Marx, Werner; Bornmann, Lutz; Barth, Andreas; Leydesdorff, Loet: Detecting the Historical Roots of Research Fields by Reference Publication Year Spectroscopy (RPYS). In: Journal of the Association for Information Science and Technology (2014), 65(4), S. 751-764. http://dx.doi.org/doi:10.1002/asi.23089.

Marx, Werner; Bornmann, Lutz: Tracing the origin of a scientific legend by reference publication year spectroscopy (RPYS): the legend of the Darwin finches. In: Scientometrics (2014), 99(3), S. 839-844. http://dx.doi.org/doi:10.1007/s11192-013-1200-8.

Marx, Werner; Bornmann, Lutz: Change of perspective: bibliometrics from the point of view of cited references-a literature overview on approaches to the evaluation of cited references in bibliometrics. In: Scientometrics (2016), im Druck. http://dx.doi.org/doi: 10.1007/s11192-016-2111-2.

Moed, Henk F.: Citation Analysis in Research Evaluation, Dordrecht, Springer, 2005.

Pritchard, Alan: Statistical Bibliography or Bibliometrics? In: Journal of Documentation (1969), 25(4), S. 348-349.

Römpp Lexikon Chemie (2016): https://roempp.thieme.de/.

Thor, Andreas; Marx, Werner; Leydesdorff, Loet; Bornmann, Lutz: Introducing Cited References Explorer (CRExplorer): A program for Reference Publication Year Spectroscopy with Cited References Disambiguation. In: Journal of Informetrics (2016), 10(2), S. 503-515. http://dx.doi.org/10.1016/j.joi.2016.02.005. Programm und Dokumentation: http://www.crexplorer.net/.

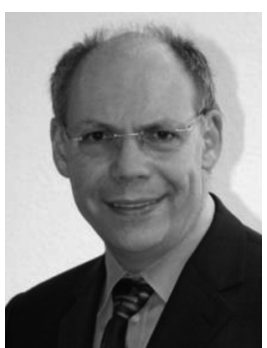

\section{Dr. Andreas Barth}

Leiter Business Development

FIZ Karlsruhe

Leibniz-Institut für Informations-

infrastruktur

Hermann-von-Helmholtz-Platz 1

76344 Eggenstein-Leopoldshafen

Telefon 07247808450

andreas.barth@fiz-karlsruhe.de

www.fiz-karlsruhe.de

orcid.org/0000-0002-1626-5121

Studium der Chemie und Physik an den Universitäten von Bonn, Freiburg und Heidelberg. Promotion zum Dr. rer.nat. am Institut für Theoretische Chemie der Universität Heidelberg. Seit 1984 am FIZ Karlsruhe zunächst in der Software-Entwicklung und dann in verschiedenen leitenden Positionen tätig. Seit einigen Jahren verantwortlich für das Business Development. Publikationen zur Quantenchemie \& Vielteilchentheorie, Information Retrieval und chemischen Datenbanken sowie zur Bibliometrie. Ein Schwerpunkt in den letzten Jahren war die Weiterentwicklung der topologischen Konzepte zum Retrieval von Markush-Strukturen.

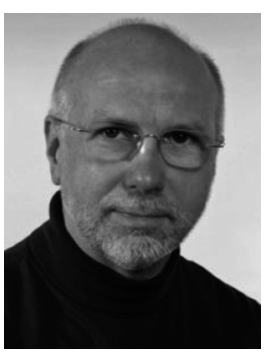

\author{
Dr. Werner Marx \\ Max-Planck-Institut für Festkörperforschung \\ Informationsservice \\ Heisenbergstraße 1 \\ 70569 Stuttgart \\ Telefon: 07116891285 \\ w.marx@fkf.mpg.de \\ http://www.fkf.mpg.de/ivs \\ orcid.org/0000-0002-1763-5753
}

Studium der Chemie an der Universität Bonn und dort Promotion über atmosphärenchemische Reaktionen im Rahmen der Umwelt- und Klimaforschung. Seit 1982 am Max-Planck-Institut für Festkörperforschung in Stuttgart. Dort bis 2014 (Ruhestand) verantwortlich für die Servicegruppe Fachinformation, einer zentralen Einrichtung zur Unterstützung der Max-Planck-Institute in Fragen der wissenschaftlichen Information und Forschungsbewertung. Seit 2014 Gastwissenschaftler am Max-Planck-Institut für Festkörperforschung. Rund 100 wissenschaftliche Publikationen im Bereich Bibliometrie und deren Einsatz in der Forschungsbewertung und Wissenschaftsgeschichte. 